\title{
A Multi-Scale Feature Recalibration Network for End-to-End Single Channel Speech Enhancement
}

\author{
Yang Xian, Student Member, IEEE, Yang Sun, Member, IEEE, \\ Wenwu Wang, Senior Member, IEEE, Syed Mohsen Naqvi, Senior Member, IEEE
}

\begin{abstract}
Deep neural networks based methods dominate recent development in single channel speech enhancement. In this paper, we propose a multi-scale feature recalibration convolutional encoder-decoder with bidirectional gated recurrent unit (BGRU) architecture for end-to-end speech enhancement. More specifically, multi-scale recalibration 2-D convolutional layers are used to extract local and contextual features from the signal. In addition, a gating mechanism is used in the recalibration network to control the information flow among the layers, which enables the scaled features to be weighted in order to retain speech and suppress noise. The fully connected layer (FC) is then employed to compress the output of the multiscale 2-D convolutional layer with a small number of neurons, thus capturing the global information and improving parameter efficiency. The BGRU layers employ forward and backward GRUs, which contain the reset, update, and output gates, to exploit the interdependency among the past, current and future frames to improve predictions. The experimental results confirm that the proposed MCGN method outperforms several state-ofthe-art methods.
\end{abstract}

Index Terms-single channel, speech enhancement, feature recalibration, multi-scale convolutional layer, bidirectional gated recurrent unit (BGRU)

\section{INTRODUCTION}

$\mathbf{T}$ HE intelligibility and quality of the speech signal recorded in a real acoustic scene are often degraded by the background noise and interfering sound in the environment. Speech enhancement aims to recover the target speech by removing the background noise and interfering sound from noisy speech mixtures. Single channel speech enhancement refers to the scenario, where only a single mixture is available, which is an extreme case of the under-determined problem, i.e. the number of sources is greater than the number of mixtures. Such a problem can be found in many real-world applications, such as mobile communication, automatic speech recognition, and robotics [1]-[5].

A wide variety of methods have been proposed for speech enhancement. Conventional methods include statistical methods, such as Wiener filtering [6] and minimum mean-square error (MMSE) estimation [7], based on statistical modelling of spatial, spectral, or temporal features derived from the

Y. Xian and S. M. Naqvi are with the Intelligent Sensing and Communications Research Group, School of Engineering, Newcastle University, Newcastle upon Tyne NE1 7RU, U.K. (e-mails: Y.Xian2@newcastle.ac.uk; Mohsen.Naqvi@newcastle.ac.uk)

Y. Sun is with Big Data Institute, University of Oxford, Oxford, OX3 7LF, U.K.(e-mail: Yang.sun@bdi.ox.ac.uk)

W. Wang is with the Center for Vision Speech and Signal Processing, Department of Electrical and Electronic Engineering, University of Surrey, Surrey GU2 7XH, U.K. (e-mail: W.Wang@ surrey.ac.uk) sensor signals. For instance, the MMSE estimator achieves speech enhancement by modeling the speech and noise spectral components as statistically independent Gaussian random variables.

Deep neural networks (DNNs) are today considered stateof-the-art in speech enhancement. Unlike the conventional methods, the DNNs based methods [8]-[10] aim to learn a mapping or masking relationship between the representations of noisy speech mixture and target speech, via a training process. Then, the trained model is used to make prediction of the target speech directly (via mapping) [11], or the T-F mask (via masking) [12]-[14], where either ideal binary mask (IBM) or ideal ratio mask (IRM) has been used as the training target. Recent results show that the mapping based methods outperform the masking based methods [15].

Different from vanilla DNNs, recurrent neural network (RNN) has been used for temporal modelling of speech and offers advantages in mismatched conditions [16]. In particular, long short-term memory (LSTM) [17] employs the cell memory, input, output and forget gates to capture the interdependency between the past and current frames, which improves the accuracy of the estimation for the mask and mapping relations [18]. Previous results show that it improves enhancement performance in the case of unseen speakers [15], [16]. As an extension to LSTM, the bidirectional LSTM has been proposed to also considers the impact of the future frames, thus capturing the long-term interdependency among the past, current and future frames [15].

Another promising direction has been on the exploitation of convolutional neural network (CNN), such as [19], where a convolutional encoder decoder (CED) is introduced to estimate the mapping relation between the noisy mixture and target speech. This is further improved for learning multi-resolution features, with a multi-resolution convolutional auto-encoders (MCARE) model [20], learning with dilated convolution to enlarge the receptive fields of the network in Wavenet, and learning with a gated mechanism to control the information flow among each layer [21]. Furthermore, the gated recurrent network (GRN) method is used with dilated 2-D convolutional layers to enlarge the receptive fields in the time-frequency ( $\mathrm{T}$ F) domain [15].

The recurrent and convolutional architectures have been used together to further improve enhancement performance. For example, in the convolutional recurrent network (CRN) [22], the convolutional encoder-decoder is integrated with the LSTM, where the CED is used to capture the local T-F patterns, and the LSTM is used to capture long-term 
interdependency [22]. The CRN method was shown to perform better than the LSTM.

All the above methods are supervised methods where class labels are required for training the model. In contrast, unsupervised methods have also been proposed for speech enhancement without the requirement of class labels. A wellknown method is the speech enhancement generative adversarial network (SEGAN) method [23].

The aforementioned methods are promising and represent current state-of-the-art. However, there are still several limitations. For the CED and CRN methods, a fixed kernel (filter) size is often used. The local information (i.e. feature) in the signal can be extracted by using a kernel of small size, while the contextual feature needs to be extracted with a larger kernel size. A method that can extract both local and contextual information is desired. In the LSTM and CRN models, causal systems are often designed by considering only current and past samples from the signal. However, in terms of [21], the prediction performance of the model can be further improved by considering the future samples. Therefore, in our work, the future information (i.e. a non-causal system) is considered to improve the enhancement performance.

In addition, the implementation of LSTM often involves computational loads for calculating the input, output, forget gates and cell memory [17], [24], sometimes, this can be problematic when the models are deployed on resource-limited devices. It would be desirable to use more efficient RNN models such as GRU/BGRU, with performance comparable to LSTM/BLSTM but less memory requirements. In addition, in the Inception network [25], the features of different scales are concatenated directly, and they are assigned with the equal weight. This means that features are considered as equally important, which may be problematic especially when the features are induced by noise. This could be further improved by assigning features with different weights, as shown in our work.

In this paper, we propose a multi-scale feature recalibration convolutional bidirectional GRU network (MCGN), with following specific contributions.

First, we introduce a multi-scale feature recalibration (MCFR) convolutional encoder-decoder module, where the kernels with different sizes are exploited in each convolutional layer, to obtain features in different scales. This helps capture the interdependency between the local and contextual information within the signal, and allows the feature in each scale to be assigned with a different weight in order to retain the components from speech while suppressing the components from noise.

Second, the bottleneck convolutional layers are introduced, which uses the 1-D convolutional layer with kernels of size $(1,1)$ to compress the information flow inside the proposed MCGN.

Third, connection layers are used in MCGN, including fully connected (FC) layer and BGRU layers. The FC layer is exploited to reduce the dimension of encoder output. The BGRU layers can capture the inter-dependencies among the past, current and future temporal frames. Compared with
BLSTM, they offer similar performance but require fewer parameters.

Fourth, the multi-scale convolutional output layer is proposed to accelerate the convergence. The output layer enables the enhanced output with access to the different scale convolutional operators, which facilitate network training.

The remainder of the paper is organized as follows. Section II describes the proposed MCGN method. The experimental settings and results are discussed in Section III. Section IV states the conclusions.

\section{The Proposed Method}

\section{A. Problem Statement}

In single channel speech enhancement, the noisy speech mixture can be written as:

$$
y(m)=s(m)+n(m)
$$

where $y(m)$ denotes the noisy speech, $s(m)$ and $n(m)$ represent the clean speech signal and noise at discrete time $m$, respectively. By using the short-time Fourier Transform (STFT), the noisy speech mixture at time frame $t \in[1,2, . ., T-1, T]$ and frequency bin $f \in[1,2, . ., F-1, F]$ is represented as:

$$
Y_{t, f}=S_{t, f}+N_{t, f}
$$

where $S_{t, f}$ and $N_{t, f}$ are the STFT of the clean speech signal and noise, respectively. The neural network model is trained to find the mapping relation $G_{\theta}$ between the magnitude spectrum of the clean speech signal $\left|S_{t, f}\right|$ and the noisy speech mixture $\left|Y_{t, f}\right|, G_{\theta}$ is parametrized by $\theta$. The mapping function is estimated by optimizing the loss function as:

$$
\begin{aligned}
\text { Loss } & =\frac{1}{T F} \sum_{t=1}^{T} \sum_{f=1}^{F}\left[G_{\theta}\left(\left|Y_{t, f}\right|\right)-\left|S_{t, f}\right|\right]^{2} \\
& =\frac{1}{T F} \sum_{t=1}^{T} \sum_{f=1}^{F}\left(\left|\hat{S}_{t, f}\right|-\left|S_{t, f}\right|\right)^{2}
\end{aligned}
$$

where $\left|\hat{S}_{t, f}\right|$ is the magnitude spectrum of the estimated target speech, which is combined with phase information of the noisy mixture to recover the target speech.

\section{B. Proposed Network Architecture}

The details of the proposed MCGN architecture are shown in Fig. 1. The MCGN contains four parts, i.e. convolutional encoder, convolutional decoder, connection layers, and multiscale convolutional output layers. The magnitude spectrum of the noisy mixture is fed to the proposed MCGN, which outputs the estimated magnitude spectrum of the target speech. The convolutional encoder consists of six convolutional layers containing four multi-scale convolutional layers, an input convolutional (the first) layer and a bottleneck convolutional layer. The multi-scale convolutional layers contain five sub convolutional blocks with varied kernel sizes. Similarly, the convolutional decoder has a symmetric structure with the 


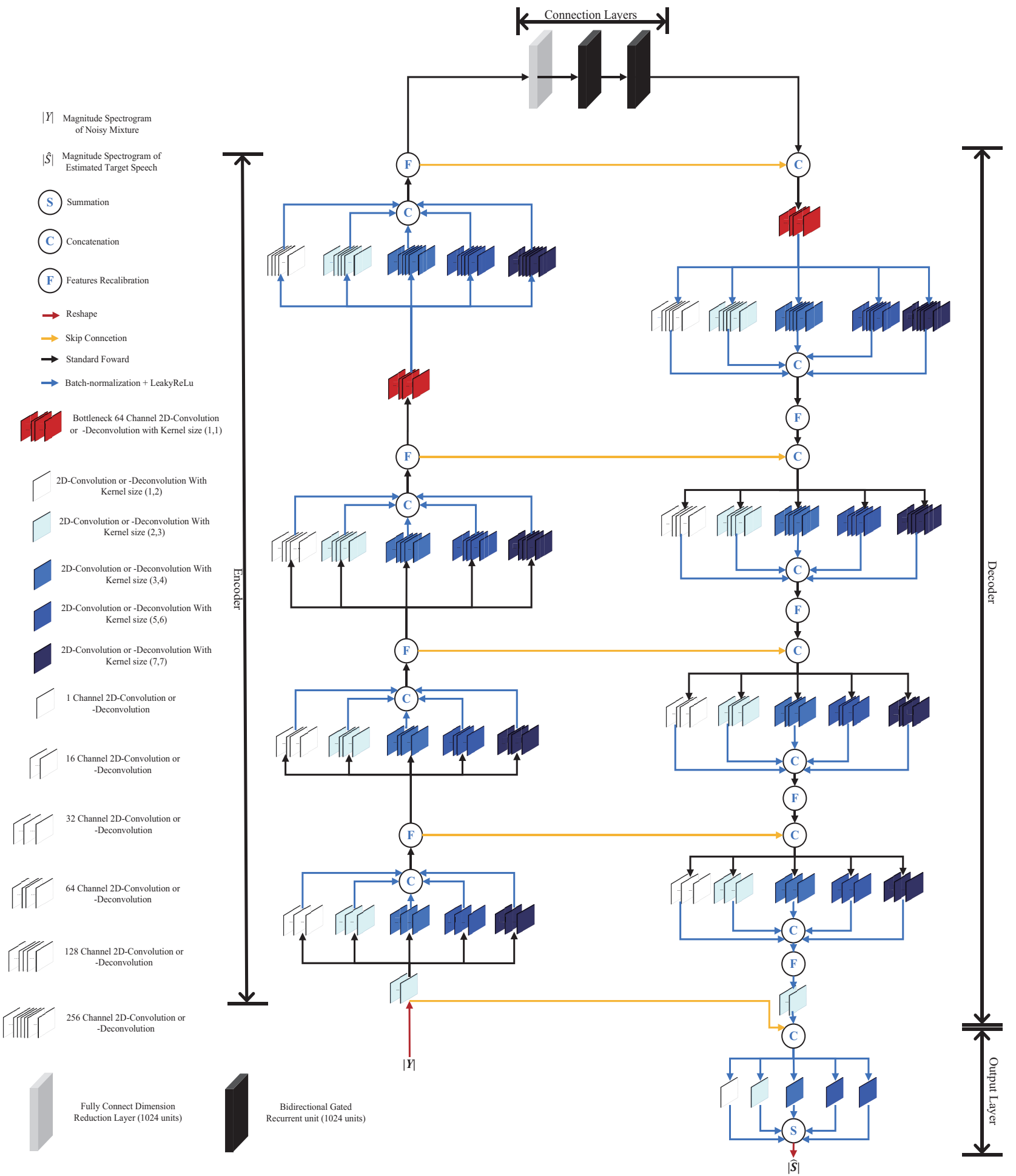

Fig. 1. The architecture of the proposed MCGN. The components and their functions are shown at the left of the figure. The overlapped 3-D boxes represent the multi-channel 2-D convolutional neural networks. The colored arrows and named circles represent the information flow and operations. The convolutional encoder is on the left of the figure, and the convolutional decoder is on the right of the figure, connection layers are shown in the middle of figure. The figure is color-coded to facilitate understanding. 
convolutional encoder. The output of the convolutional encoder is fed to the connection layers. After processed by the connection layers, the information flow is fed to the convolutional decoder. In addition, the skip connections are added among the convolutional encoder and decoder. The layer hyper-parameters can be found in Fig. 1. The stride size of all layers is $(1,2)$, except the multi-scale output layer, which has a fixed stride size $(1,1)$.

\section{Multi-Scale Feature Recalibration Convolutional Layer}

The receptive field is a region where $\mathrm{CNN}$ can affect a particular high-level feature. A small receptive field is feasible to extract local information, and a large receptive field offers contextual information [15]. In conventional CNN, a fixed kernel size is often used, as a result, it compromises between local and contextual information extracted from the signal. To address this limitation, a multi-scale convolutional feature recalibration (MCFR) layer is designed to capture the information on different scales and generate the multiscaled feature. As shown in Fig. 2, MCFR contains several convolutional operators, which use the kernels of different sizes to capture the information with various scales. The convolutional operators with the small kernel sizes can extract the feature from the short duration speech, thus capturing the adjacent T-F points local dependency. The smallest kernel size $(1,2)$ is employed, which allows the feature from two adjacent $\mathrm{T}-\mathrm{F}$ points to be extracted. The convolutional operators with large kernel sizes offer large receptive fields and can extract features from long-duration speech. These features contain contextual information compared with the feature extracted by kernels with smaller sizes. The batch-normalization is used after each convolutional operator. Different from the standard CNN, which uses the ReLU activation function [26], our proposed MCGN utilizes the activation function LeakyReLU [27]. Then, we concatenate the outputs of each convolutional operator into a single output vector, forming the input of the next stage, as shown in Fig. 2. The multi-scale decovolutional layer has a similar structure as the one in MCFR, by replacing the convolutional operators with deconvolutional operators.

After the features at different scales are extracted by using the convolutional operators with varied kernel sizes, a feature recalibration module is introduced to help the network to be selective when using these scaled features, i.e. by assigning different weights to features. It is shown on the bottom of Fig. 2. We refer to the proposed multi-scale convolutional feature recalibration layer as the MCFR layer. In the MCFR layer, we use $n$ sub-convolutional blocks, and each block has the same number of channels but different kernel sizes to capture the features in different scales. The input of the multi-scale layer is $\mathbf{X}$, and the output is $\mathbf{K}=\left[\mathbf{k}_{1}, \mathbf{k}_{2}, . ., \mathbf{k}_{n}\right]$, where $\mathbf{k}_{n}$ is captured by the $n$-th sub 2-D convolutional block that has different kernel size compared with other 2-D convolutional blocks.

There are several operations for estimating the recalibration coefficients, based on two criteria: the recalibration coefficient could capture the nonlinear relation inside the multiscaled feature, and allocate relatively higher weights to speech

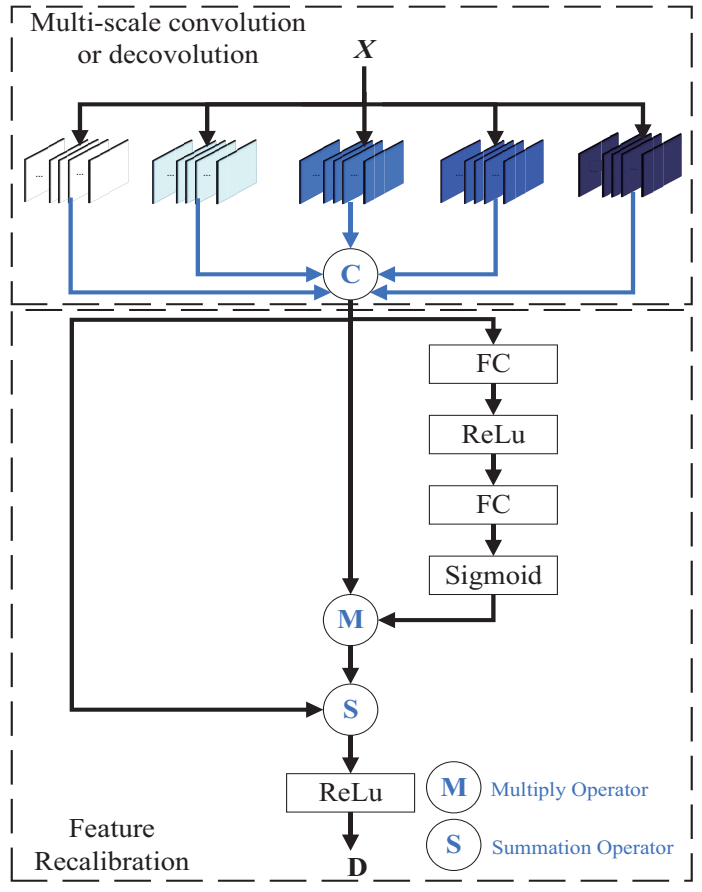

Fig. 2. Multi-scale feature recalibration network, where $\boldsymbol{X}$, and $\boldsymbol{D}$ represent the input and output of the MCFR module, respectively. The multi-scale convolution or decovolution is shown on top of the figure, the bottom of the figure shows the feature recalibration module.

components and lower weights to noise components within the feature. We use the following operations to meet these criteria: two FC layers, ReLU and Sigmoid activations. These operations are shown as follows,

$$
\begin{gathered}
\mathbf{c}_{1 n}=\mathbf{w}_{1 n} \odot \mathbf{k}_{n}+\mathbf{b}_{1 n} \\
\mathbf{a}_{n}=\max \left[0, \mathbf{c}_{1 n}\right] \\
\mathbf{c}_{2 n}=\mathbf{w}_{2 n} \odot \mathbf{a}_{n}+\mathbf{b}_{2 n} \\
\mathbf{r s}_{n}=\frac{e^{\mathbf{c}_{2 n}}}{e^{\mathbf{c}_{2 n}}+\mathbf{j}}
\end{gathered}
$$

where $\mathbf{w}_{1 n}, \mathbf{w}_{2 n}$ denote the weight parameters, $\odot$ denotes element-wise multiplication, $\mathbf{b}_{1 n}, \mathbf{b}_{2 n}$ represent the biases. $\mathbf{c}_{1 n}$ and $\mathbf{c}_{2 n}$ represent the operations in FC1 and FC2 layers, respectively. $\mathbf{j}=[1,1, \ldots, 1]$, and it has the same dimension as $\mathbf{c}_{2 n}$. The exponential function $e$ is operated element-wise on $\mathbf{c}_{2 n}$, so is the division in the right hand side of equation (7). The vector $\mathbf{r s}_{n}$ contains the recalibration coefficient of the $n$-th scaled feature. Empirically, we opt for the ReLU function as (5), which is employed as a non-negative constraint. Inspired by the success of the gating mechanism, we introduce Sigmoid as a gating function to control the information flow, which aims to assign different weights to speech and noise components. The rescaled $n$-th feature is:

$$
\mathbf{p}_{n}=\mathbf{k}_{n} \odot \mathbf{r s}_{n}
$$

Therefore, the rescaled multi-scale feature is $\mathbf{P}=$ $\left[\mathbf{p}_{1}, \mathbf{p}_{2}, \ldots, \mathbf{p}_{n}\right]$. We introduce deep skip connection (as in residual learning [28]) inside the MCFR layer. In addition, the residual learning does not introduce any additional parameters. 
Mathematically, the original relation for the MCFR layer is $\mathbf{D}=\mathbf{P}$, by using the residual learning and the ReLU function, the relation becomes:

$$
\mathbf{D}=\max [0, \mathbf{K}+\mathbf{P}]
$$

Following the extraction of multi-scale features, the proposed MCGN learns the weights and applies them to these features which help retain speech components and suppresses the noise components in the noisy mixture.

\section{Bottlenecks Convolutioal Layers}

One of the practical problems in multi-scale convolutional layers that need to be solved is the concatenation of the multi-scale features, which would increase the dimension of the features and cause an increase in computational cost. Therefore, a structure that can retain the information while reducing the complexity (e.g. dimension) is needed. Inspired by the embedding techniques that a low dimensional embedding might contain sufficient information about a relatively large patch [25], [29], we introduce the bottleneck convolutional layers in the proposed MCGN architecture. The bottleneck convolutional layer is a 2-D convolutional layer with $(1,1)$ kernels and 64 channels, followed by the batch-normalization and LeakeyReLU [27]. It is located before the last convolutional encoder layer and the first decoder layer, as shown in Fig. 1 (red convolutional blocks). The first bottleneck convolutional layer reduces the dimension from 640-D to 64-D for the last encoder layer, and the second bottleneck convolutional layer reduces the dimension from 128-D to 64-D for the first decoder layer.

\section{E. Connection Layers}

The original convolutional encoder-decoder does not well utilized the long-term temporal information, which, nevertheless, may be valuable in speech enhancement [16], [22]. The CRN method uses the LSTM to capture the long-term interdependency between the past and current temporal frames. However, CRN is designed for the casual problem, which utilizes long-term interdependency between past and current temporal frames. According to [21], the future frames could be used to improve enhancement performance. In our work, we introduce BGRU to capture the long-term interdependency among the past, current and future temporal frames. In comparison, GRU offers comparable performance to LSTM [24], [30], [31], but has an advantage in parameter efficiency. However, the merging of the multi-scaled convolutional sub-blocks would lead to an inevitable increase in its dimension. Therefore, it is necessary to find a way to retain the information and, at the same time, to reduce the dimension and computational cost. To address this, we use a fully connected (FC) layer, as the number of parameters of the fully connected dense layer is smaller than that of the RNN based layer, leading to a reduced dimension in the output of the FC layer, as compared with the output of the encoder.

\section{F. Multi-Scale Output Layer}

We add the skip connection from the input to the multiscale output layer, as shown at the bottom of Fig. 1. As a result, the multi-scale output layer can estimate the magnitude of the target speech from the previous layer's information flow and the input magnitude of the noisy mixture. The multi-scale output layer is a 2-D deconvolutional layer, which contains five sub-blocks, and the kernel sizes of these sub-layers are different. Unlike the MCFR layer, these varying scaled features are concatenated, the different scaled features are summed together to generate an output matrix with the same size as the input matrix. Thus, the multi-scale output layer utilizes local and contextual information. The stride size of the output layer is set to $(1,1)$. Batch-normalization and linear activation are followed.

\section{EXPERIMENTAL EVALUATIONS}

\section{A. Datasets}

We evaluate our system with three experiments using three different datasets. In the first experiment, we use 1000 clean utterances mixed with 20 noise signals to generate the training set in our first experiment. The clean utterances are randomly selected from the TIMIT corpus [32], and noise files are selected from Non-Speech Sounds [33] and NOISEX-92 [34] datasets. Similarly, 100 clean utterances are mixed with 6 noise signals to generate the testing datasets. To better evaluate enhancement performance, the speakers in the training set are different from the speakers in the testing dataset. Meanwhile, the testing noisy interferences are categorized into two types, the seen noises (Babble, Leopard, F16) and the unseen noises (N56, N72, White). Babble, Leopard, F16, N56, N72 are nonstationary noises, and White is stationary noise. N56 and N72 are wind and water sounds, respectively. The noisy mixtures are generated by mixing the clean utterances and noises at $5 \mathrm{~dB}, 0 \mathrm{~dB}$ and $5 \mathrm{~dB}$ signal-to-noise ratio (SNR) levels. In total, about 50 hours $(3 \times 3 \times 1000 \times 20 \div 3600)$ noisy mixtures are used to train the networks.

In the second experiment, we evaluate the proposed method on a published dataset [21], [23]. The datasets are generated by using the VCTK corpus [35] and Environment Multichannel Acoustic Noise Database [36]. The utterances from 28 speakers and 2 speakers are used for training and testing, respectively. Each speaker has spoken around 400 sentences. The training utterances are mixed with 10 types of noise in four SNR levels $(0 \mathrm{~dB}, 5 \mathrm{~dB}, 10 \mathrm{~dB}$ and $15 \mathrm{~dB})$. In total, there are 11,572 noisy mixtures for training. Similarly, the testing utterances are mixed with 5 types of noise in four SNR levels $(2.5 \mathrm{~dB}, 7.5 \mathrm{~dB}, 12.5 \mathrm{~dB}$ and $17.5 \mathrm{~dB})$. In total, the testing set includes 824 noisy mixtures, where both the speakers and noises are unseen in the training set.

In the third experiment, we evaluate the proposed MCGN method with a larger dataset. For the training set, we randomly select 2500 clean utterances from the TIMIT [32] and VCTK [35] corpora, mix them with 20 different noise signals selected from the Non-Speech Sounds [33] and NOISEX-92 [34] datasets, to generate 50000 training mixtures for each SNR level $(-5 \mathrm{~dB}, 0 \mathrm{~dB}$, and $5 \mathrm{~dB})$. Similarly, for the testing set, 
TABLE I

SPEECH ENHANCEMENT PERFORMANCE COMPARISONS IN TERMS OF STOI AND PESQ OVER THREE DIFFERENT TYPES OF SEEN NOISES WITH DIFFERENT BASELINE METHODS AND SNR LEVELS. EACH RESULT IS THE AVERAGE VALUE OF 100 EXPERIMENTS. Italic TEXT REFERS TO THE PROPOSED METHODS. BOLD NUMBER INDICATES THE BEST PERFORMANCE.

\begin{tabular}{|c|c|c|c|c|c|c|c|c|c|c|c|c|}
\hline Measure & \multicolumn{12}{|c|}{ STOI (\%) } \\
\hline Noises & \multicolumn{4}{|c|}{ Babble } & \multicolumn{4}{|c|}{ Leopard } & \multicolumn{4}{|c|}{ F16 } \\
\hline SNR & $-5 \mathrm{~dB}$ & $0 \mathrm{~dB}$ & $5 \mathrm{~dB}$ & Avg. & $-5 \mathrm{~dB}$ & $0 \mathrm{~dB}$ & $5 \mathrm{~dB}$ & Avg. & $-5 \mathrm{~dB}$ & $0 \mathrm{~dB}$ & $5 \mathrm{~dB}$ & Avg. \\
\hline Noisy Mixture & 53.86 & 63.05 & 71.66 & 62.86 & 71.68 & 75.57 & 78.92 & 75.39 & 54.39 & 64.07 & 73.37 & 63.94 \\
\hline DNN [11] & 66.36 & 72.91 & 79.26 & 72.83 & 77.11 & 80.55 & 83.26 & 80.30 & 69.17 & 76.24 & 81.35 & 78.79 \\
\hline S-DNN [10] & 66.80 & 73.66 & 79.63 & 73.36 & 78.34 & 81.54 & 83.98 & 81.29 & 69.77 & 76.46 & 81.86 & 75.97 \\
\hline LSTM [16] & 68.78 & 75.81 & 81.54 & 75.38 & 80.76 & 83.32 & 85.40 & 83.16 & 72.13 & 77.86 & 83.39 & 77.79 \\
\hline BLSTM [15] & 69.30 & 76.63 & 82.04 & 75.99 & 81.10 & 83.85 & 85.58 & 83.59 & 72.19 & 78.15 & 83.61 & 77.98 \\
\hline MRCAE [20] & 65.92 & 72.83 & 78.85 & 72.53 & 77.50 & 80.56 & 83.09 & 81.25 & 69.10 & 75.51 & 80.88 & 75.16 \\
\hline CRN [22] & 70.10 & 76.95 & 81.88 & 76.31 & 81.20 & 84.02 & 85.80 & 83.67 & 72.65 & 78.98 & 83.90 & 78.51 \\
\hline CRBN & 70.30 & 77.08 & 81.96 & 76.45 & 81.20 & 84.20 & 85.90 & 83.77 & 73.49 & 79.12 & 84.14 & 78.92 \\
\hline GRN [15] & 71.60 & 77.08 & 82.21 & 76.94 & 82.20 & 84.15 & 86.08 & 84.14 & 72.94 & 79.27 & 83.56 & 78.59 \\
\hline$M C G N$ & 75.02 & 80.52 & 84.43 & 79.99 & 84.10 & 85.78 & 87.31 & 86.55 & 77.49 & 81.56 & 85.60 & 81.55 \\
\hline Measure & \multicolumn{12}{|c|}{ PESQ } \\
\hline Noises & \multicolumn{4}{|c|}{ Babble } & \multicolumn{4}{|c|}{ Leopard } & \multicolumn{4}{|c|}{ F16 } \\
\hline SNR & $-5 \mathrm{~dB}$ & $0 \mathrm{~dB}$ & $5 \mathrm{~dB}$ & Avg. & $-5 \mathrm{~dB}$ & $0 \mathrm{~dB}$ & $5 \mathrm{~dB}$ & Avg. & $-5 \mathrm{~dB}$ & $0 \mathrm{~dB}$ & $5 \mathrm{~dB}$ & Avg. \\
\hline Noisy Mixture & 1.28 & 1.52 & 1.81 & 1.53 & 1.75 & 1.99 & 2.22 & 1.97 & 1.31 & 1.54 & 1.83 & 1.56 \\
\hline DNN [11] & 1.58 & 1.90 & 2.20 & 1.89 & 2.03 & 2.31 & 2.50 & 2.28 & 1.73 & 2.08 & 2.31 & 2.04 \\
\hline S-DNN [10] & 1.69 & 2.00 & 2.28 & 1.99 & 2.25 & 2.45 & 2.67 & 2.46 & 1.82 & 2.11 & 2.35 & 2.09 \\
\hline LSTM [16] & 1.82 & 2.15 & 2.44 & 2.14 & 2.41 & 2.61 & 2.80 & 2.61 & 1.97 & 2.28 & 2.52 & 2.25 \\
\hline BLSTM [15] & 1.84 & 2.19 & 2.47 & 2.16 & 2.44 & 2.67 & 2.84 & 2.65 & 2.02 & 2.30 & 2.54 & 2.29 \\
\hline MRCAE [20] & 1.72 & 2.04 & 2.31 & 2.02 & 2.25 & 2.47 & 2.69 & 2.47 & 1.85 & 2.15 & 2.39 & 2.13 \\
\hline CRN [22] & 1.91 & 2.22 & 2.49 & 2.21 & 2.50 & 2.70 & 2.90 & 2.69 & 2.02 & 2.30 & 2.54 & 2.29 \\
\hline CRBN & 1.93 & 2.23 & 2.50 & 2.22 & 2.51 & 2.72 & 2.90 & 2.71 & 2.09 & 2.38 & 2.58 & 2.35 \\
\hline GRN [15] & 1.94 & 2.24 & 2.49 & 2.22 & 2.53 & 2.73 & 2.92 & 2.73 & 2.05 & 2.34 & 2.58 & 2.32 \\
\hline$M C G N$ & 2.16 & 2.43 & 2.65 & 2.41 & 2.70 & 2.88 & 3.04 & 2.87 & 2.23 & 2.47 & 2.70 & 2.47 \\
\hline
\end{tabular}

we randomly select 500 clean utterances and mix them with 5 different noise signals, to generate 2500 noisy mixtures for each SNR level. The speakers of the training dataset are different from those in the testing dataset. The Babble, Leopard, F16 are seen noises, while N56 and N72 are unseen noises.

The signal to distortion ratio improvement ( $\Delta$ SDR) [37], perceptual evaluation of speech quality (PESQ) [38] and shorttime objective intelligibility (STOI) [39] are used to measure the performance. The $\Delta \mathrm{SDR}$ is equal to the SDR of the estimated speech minus the SDR of the unprocessed noisy mixture. The PESQ ranges from -0.5 to 4.5 , which indicates the speech perception quality score. The STOI ranges from zero to one, which indicates the intelligibility quality of human speech. The higher values of the measurements indicate better enhancement performance.

\section{B. Baselines and Parameters}

The proposed MCGN is compared with seven baseline methods, including the standard DNN method from [11], the DNN method with skip connection S-DNN from [10], the LSTM model used in [16], the BLSTM model used in [15], the CNN based methods, the MRCAE method from [20], and the GRN method in [15]. The parameters of the CRN model are set by following [22]. LSTM and BLSTM have four hidden layers, where each hidden layer contains 1024 units with a dropout rate of 0.2 , and the output layer is a dense layer. The MRCAE is a five-layered 1-D convolutional encoder decoder. The encoder consists of two multi-resolution 1-D convolutional layers, and the decoder mirrors the encoder. A deconvolutional layer is used as the output layer of MRCAE. The CRN consists of the 2-D convolutional encoder, twolayered LSTM and 2-D convolutional decoder, which are connected by standard feed-forward connections and skip
TABLE II

THE $p$-VALUE OF THE T-TEST AT 5\% SIGNIFICANCE LEVEL, BETWEEN THE PROPOSED METHOD AND THE BASELINE METHODS. $H_{0}$ DENOTES THE NULL HYPOTHESIS, AND (+) INDICATES THAT THE DIFFERENCE AMONG THE PAIR IS STATISTICALLY SIGNIFICANT AT THE $95 \%$ CONFIDENCE LEVEL.

\begin{tabular}{cclccc}
\hline \hline Measures & \multicolumn{2}{c}{ STOI } & \multicolumn{2}{c}{ PESQ } \\
\hline & $p$-value & $H_{0}$ & & $p$-value & $H_{0}$ \\
\cline { 2 - 3 } \cline { 4 - 6 } Noisy & $1.49 \mathrm{E}-05$ & $(+)$ & & 4 E -12 & $(+)$ \\
DNN [11] & $5.22 \mathrm{E}-06$ & $(+)$ & & $1.76 \mathrm{E}-07$ & $(+)$ \\
S-DNN [10] & $1.08 \mathrm{E}-05$ & $(+)$ & & $3.20 \mathrm{E}-09$ & $(+)$ \\
LSTM [16] & $8.16 \mathrm{E}-05$ & $(+)$ & & $3.49 \mathrm{E}-07$ & $(+)$ \\
BLSTM [15] & $1.93 \mathrm{E}-04$ & $(+)$ & & $1.46 \mathrm{E}-06$ & $(+)$ \\
MRCAE [20] & $3.19 \mathrm{E}-06$ & $(+)$ & & $1.87 \mathrm{E}-06$ & $(+)$ \\
CRN [22] & $1.44 \mathrm{E}-04$ & $(+)$ & & $2.74 \mathrm{E}-07$ & $(+)$ \\
CRBN & $7.71 \mathrm{E}-05$ & $(+)$ & & $1.13 \mathrm{E}-05$ & $(+)$ \\
GRN [15] & $1.01 \mathrm{E}-04$ & $(+)$ & & $1.08 \mathrm{E}-06$ & $(+)$ \\
\hline \hline
\end{tabular}

connections. The GRN is a 62-layered fully connected dilated convolutional neural network with the residual. The aforementioned baseline methods and proposed MCGN method take the STFT magnitude spectrum of the noisy speech mixture as the input features, and output the corresponding magnitude spectrum of the estimated target speech. The estimated magnitude spectrum is combined with the noisy phase to resynthesize the estimated target speech waveform. Furthermore, the proposed MCGN model trained on the published dataset [21], [23] is compared with the SEGAN and Wavenet. The SEGAN employs generator and discriminator to learn and judge the input data distribution, which uses the adversarial training [23]. The Wavenet is a 30-layered fully connected convolutional neural network [21].

The input and output layers for all methods contain 257 units. The baseline methods and proposed MCGN method are trained with the Adam optimization algorithm [40]. The initial learning rate is set to 0.0001 . The mean square error (MSE) is employed as the objective function for the baseline and the proposed MCGN methods. The dropout rate is fixed to 

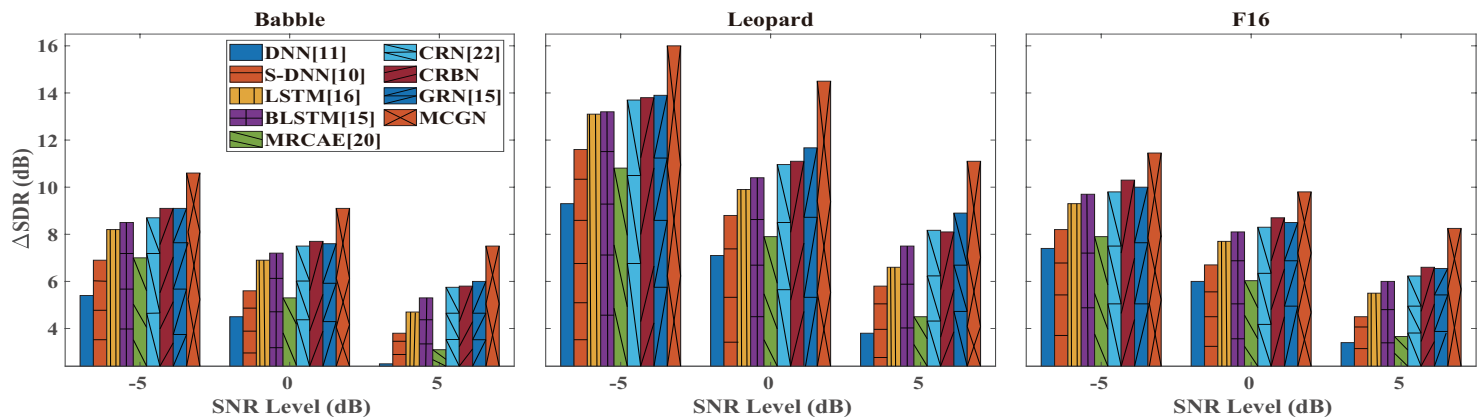

Fig. 3. Speech enhancement performance comparison in terms of $\Delta$ SDR for three types of seen noises with different methods and SNR levels. Each result is the averaged value of 100 independent experiments.
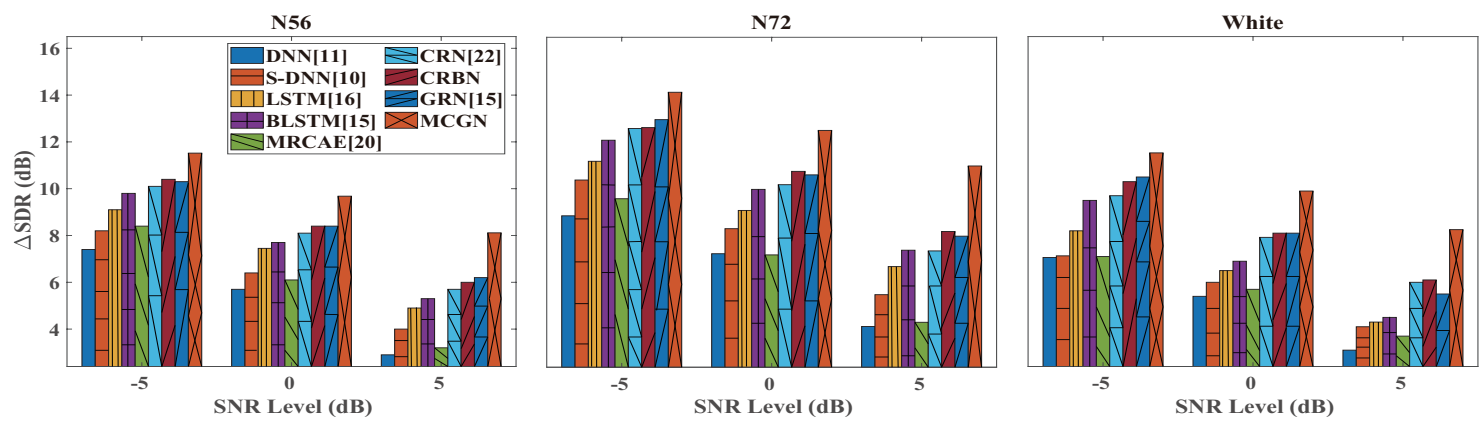

Fig. 4. Speech enhancement performance comparison in terms of $\Delta$ SDR for three unseen noises with different methods and SNR levels. Each result is the averaged value of 100 experiments.

0.2 . The sample rate of noisy speech mixtures is $16 \mathrm{kHz}$, and the window length is 512 . The time resolution is $32 \mathrm{~ms}$, and frequency resolution is $32.15 \mathrm{~Hz}$. The next two sections (i.e. Sections III.C and III.D) report the results based on the first dataset, while Section III.E and III.F present results for the second and third dataset, respectively.

\section{Unseen Speakers with Seen Noises}

Fig. 3 and Table I provide experimental results in terms of $\triangle$ SDR, STOI and PESQ for the baseline and the proposed methods with real-world noises. The speakers used in testing are unseen in the training data. The noises used in testing include Babble, Leopard, and F16.

The DNN generates, on average, $\Delta \mathrm{SDR}=5.49 \mathrm{~dB}$, STOI $=76.26 \%$ and PESQ $=2.07$, which offers the worst enhancement performance across all the compared methods. These results show that effectiveness of DNN remains insufficient. The S-DNN slightly outperforms the DNN, because S-DNN explicates the skip connection. The MRCAE method uses the multi-resolution 1-D convolutional encoder decoder and offers a small improvement over the DNN in terms of $\triangle \mathrm{SDR}$, and PESQ.

The LSTM generates, on average, $\Delta \mathrm{SDR}=8.03 \mathrm{~dB}$, STOI $=78.77 \%$ and PESQ $=2.33$, which shows advantages over the DNN, S-DNN and MRCAE. Unlike the DNN, S-DNN and MRCAE method, the LSTM exploits the memory cell to keep the hidden states from the past temporal frame. The interdependency between them are captured by the LSTM, incorporating the past and current temporal frames. The BLSTM outperforms the LSTM, due to the use of forward-LSTM and backward-LSTM in every BLSTM layer. The forwardLSTM is the same as the standard LSTM, which captures the interdependency between the past and current temporal frames. However, the backward-LSTM is fed by reverse input sequence, and thus the interdependency between current and future temporal frames is also utilized to achieve further improvement over the LSTM.

The CRN obtains, on average, $\Delta \mathrm{SDR}=8.81 \mathrm{~dB}$, STOI $=$ $79.49 \%$ and $\mathrm{PESQ}=2.39$, which provides more significant improvements over the DNN, S-DNN and LSTM methods. Since the CRN captures local spatial patterns of the input magnitude spectrum, it can leverage the T-F structure of the magnitude spectrum. Moreover, the LSTM layers inside the CRN exploit the temporal dependency by using past and current temporal frames. In addition, we perform experiments for the non-casual version of CRN, namely CRBN, where the BLSTM layers replace LSTM layers. The experimental results show that the CRBN offers slight improvements over the CRN method, which confirms that the interdependency between the current and future frames improves predictions by the model. The GRN outperforms the CRN by using the dilated convolutional layers.

The proposed MCGN gets the highest improvements over the baseline methods, and it achieves, on average, $\triangle \mathrm{SDR}=$ $10.88 \mathrm{~dB}, \mathrm{STOI}=82.42 \%$ and PESQ $=2.58$, which are almost $1.7 \mathrm{~dB}, 2.53 \%$ and 0.16 higher than those achieved by the CRN method. The MCGN encodes the input magnitude spectrum in different scales. The local interdependency is captured by the convolutional sub-layers with small kernel sizes. The convolutional sub-layers with large kernel sizes are used to find the interdependency from the larger regions. By using the 
TABLE III

SPEECH ENHANCEMENT PERFORMANCE COMPARISONS IN TERMS OF STOI AND PESQ OVER THREE TYPES OF UNSEEN NOISES WITH BASELINE METHODS AND SNR LEVELS. EACH RESULT IS THE AVERAGED VALUE OF 100 EXPERIMENTS. Italic TEXT REFERS TO THE PROPOSED METHODS. BOLD NUMBER INDICATES THE BEST PERFORMANCE.

\begin{tabular}{|c|c|c|c|c|c|c|c|c|c|c|c|c|}
\hline Measure & \multicolumn{12}{|c|}{ STOI (\%) } \\
\hline Noises & \multicolumn{4}{|c|}{ N56 } & \multicolumn{4}{|c|}{ N72 } & \multicolumn{4}{|c|}{ White } \\
\hline SNR & $-5 \mathrm{~dB}$ & $0 \mathrm{~dB}$ & $5 \mathrm{~dB}$ & Avg & $-5 \mathrm{~dB}$ & $0 \mathrm{~dB}$ & $5 \mathrm{~dB}$ & Avg. & $-5 \mathrm{~dB}$ & $0 \mathrm{~dB}$ & $5 \mathrm{~dB}$ & Avg. \\
\hline Noisy Mixture & 57.07 & 68.26 & 78.37 & 67.90 & 70.87 & 76.77 & 81.63 & 76.42 & 53.40 & 62.57 & 72.32 & 62.76 \\
\hline DNN [11] & 72.59 & 78.74 & 84.07 & 78.46 & 75.34 & 81.43 & 85.00 & 80.59 & 62.73 & 70.42 & 77.32 & 70.16 \\
\hline S-DNN [10] & 72.79 & 78.74 & 84.31 & 78.85 & 76.87 & 82.18 & 85.67 & 81.57 & 63.00 & 70.63 & 77.87 & 70.50 \\
\hline LSTM [16] & 76.99 & 79.45 & 86.47 & 80.97 & 76.83 & 82.95 & 86.80 & 82.19 & 67.71 & 75.91 & 81.53 & 75.05 \\
\hline BLSTM [15] & 77.64 & 82.49 & 86.87 & 82.33 & 78.05 & 83.50 & 87.12 & 82.89 & 72.93 & 76.43 & 83.91 & 77.76 \\
\hline MRCAE [20] & 72.74 & 78.95 & 83.78 & 78.49 & 75.47 & 80.57 & 84.72 & 80.25 & 65.12 & 71.36 & 76.31 & 70.93 \\
\hline CRN [22] & 77.88 & 83.37 & 87.09 & 82.78 & 78.55 & 84.12 & 87.24 & 83.30 & 72.90 & 78.93 & 84.34 & 78.72 \\
\hline CRBN & 78.95 & 83.85 & 87.30 & 83.37 & 79.26 & 84.37 & 87.64 & 83.76 & 76.24 & 81.30 & 85.16 & 80.92 \\
\hline GRN [15] & 78.19 & 83.60 & 87.33 & 83.04 & 78.96 & 84.27 & 87.60 & 83.61 & 76.31 & 80.80 & 84.59 & 80.57 \\
\hline$M C G N$ & 82.83 & 86.85 & 89.82 & 86.50 & 81.07 & 85.52 & 88.36 & 84.98 & 78.26 & 83.81 & 87.75 & 83.27 \\
\hline Measure & \multicolumn{12}{|c|}{ PESQ } \\
\hline Noises & \multicolumn{4}{|c|}{ N56 } & \multicolumn{4}{|c|}{ N72 } & \multicolumn{4}{|c|}{ White } \\
\hline SNR & $-5 \mathrm{~dB}$ & $0 \mathrm{~dB}$ & $5 \mathrm{~dB}$ & Avg. & $-5 \mathrm{~dB}$ & OdB & $5 \mathrm{~dB}$ & Avg. & $-5 \mathrm{~dB}$ & OdB & $5 \mathrm{~dB}$ & Avg. \\
\hline Noisy Mixture & 1.14 & 1.31 & 1.57 & 1.34 & 1.58 & 1.83 & 2.06 & 1.83 & 1.04 & 1.21 & 1.47 & 1.24 \\
\hline DNN [11] & 1.69 & 1.98 & 2.16 & 1.95 & 1.77 & 2.05 & 2.23 & 2.02 & 1.32 & 1.61 & 1.87 & 1.60 \\
\hline S-DNN [10] & 1.74 & 2.05 & 2.20 & 1.98 & 1.86 & 2.11 & 2.32 & 2.10 & 1.34 & 1.69 & 1.94 & 1.66 \\
\hline LSTM [16] & 1.93 & 2.17 & 2.36 & 2.16 & 1.87 & 2.14 & 2.40 & 2.14 & 1.59 & 1.96 & 2.26 & 1.94 \\
\hline BLSTM [15] & 1.97 & 2.20 & 2.39 & 2.18 & 1.92 & 2.20 & 2.45 & 2.19 & 1.81 & 2.19 & 2.45 & 2.15 \\
\hline MRCAE [20] & 1.83 & 2.06 & 2.22 & 2.04 & 1.93 & 2.16 & 2.35 & 2.15 & 1.60 & 1.85 & 2.09 & 1.85 \\
\hline CRN [22] & 1.92 & 2.22 & 2.49 & 2.21 & 1.98 & 2.20 & 2.41 & 2.20 & 1.90 & 2.21 & 2.48 & 2.20 \\
\hline CRBN & 2.05 & 2.27 & 2.44 & 2.25 & 1.99 & 2.25 & 2.47 & 2.24 & 2.04 & 2.28 & 2.54 & 2.29 \\
\hline GRN [15] & 2.01 & 2.24 & 2.43 & 2.23 & 2.01 & 2.25 & 2.53 & 2.26 & 2.14 & 2.35 & 2.56 & 2.35 \\
\hline$M C G N$ & 2.22 & 2.40 & 2.58 & 2.40 & 2.14 & 2.40 & 2.63 & 2.39 & 2.24 & 2.55 & 2.84 & 2.54 \\
\hline
\end{tabular}

TABLE IV

THE $p$-VALUE OF THE T-TEST AT 5\% SIGNIFICANCE LEVEL, COMPARISON OF PROPOSED METHOD WITH THE BASELINE METHODS. $H_{0}$ DENOTES THE NULL HYPOTHESIS, AND $(+)$ INDICATES THE IMPROVEMENT OF TWO PAIRS IS STATISTICALLY SIGNIFICANT AT THE 95\% CONFIDENCE LEVEL.

\begin{tabular}{cccccc}
\hline \hline Measures & \multicolumn{2}{c}{ STOI } & & \multicolumn{2}{c}{ PESQ } \\
\hline & $p$-value & $H_{0}$ & & $p$-value & $H_{0}$ \\
\cline { 2 - 3 } \cline { 5 - 6 } Noisy & $1.49 \mathrm{E}-04$ & $(+)$ & & .06E-05 & $(+)$ \\
DNN [11] & $2.97 \mathrm{E}-04$ & $(+)$ & & $1.73 \mathrm{E}-05$ & $(+)$ \\
S-DNN [10] & $6.75 \mathrm{E}-04$ & $(+)$ & & $3.80 \mathrm{E}-04$ & $(+)$ \\
LSTM [16] & $4.18 \mathrm{E}-04$ & $(+)$ & & $3.05 \mathrm{E}-04$ & $(+)$ \\
BLSTM [15] & $2.24 \mathrm{E}-04$ & $(+)$ & & $4.17 \mathrm{E}-05$ & $(+)$ \\
MRCAE [20] & $9.94 \mathrm{E}-04$ & $(+)$ & & $2.42 \mathrm{E}-04$ & $(+)$ \\
CRN [22] & $1.89 \mathrm{E}-04$ & $(+)$ & & $2.62 \mathrm{E}-05$ & $(+)$ \\
CRBN & $1.06 \mathrm{E}-04$ & $(+)$ & & $1.18 \mathrm{E}-05$ & $(+)$ \\
GRN [15] & $1.94 \mathrm{E}-04$ & $(+)$ & & $2.82 \mathrm{E}-05$ & $(+)$ \\
\hline \hline
\end{tabular}

small and large size kernels, the receptive field of MCGN is enlarged, and the different scaled features are assigned with different weights. Furthermore, the BGRU layers are introduced to connect the multi-scale encoder and multi-scale decoder, which are capable of exploiting the interdependency of the past, current and future temporal frames. Besides, the raw data is fed to the output layer of the MCGN to learn the residual mapping relation.

We also perform the t-test between the proposed MCGN method and baseline methods, noisy mixtures for the unseen speakers with seen noises cases. The t-test results are shown in Table II. The $p$-values are all smaller than 0.05 and all the null hypothesis is $(+)$, which indicates that the proposed MCGN method yields a statistically significant improvement over the baseline methods.

\section{Unseen Speaker with Unseen Noises}

Fig. 4 and Table III provide experimental results in terms of $\triangle \mathrm{SDR}, \mathrm{STOI}$ and PESQ for the proposed MCGN and baseline methods with unseen noises. The testing speakers are unseen in training data. The unseen testing noises are N56, N72 and White noises.

The DNN method offers slight improvements over the noisy mixture. The MRCAE outperforms the DNN method in terms of $\triangle \mathrm{SDR}$ and PESQ, but its STOI performance is worse than that of DNN and S-DNN. These results show that the shallow structure and small channel numbers can limit the performance of MRCAE. Besides, the large size filters increase computational cost. The skip connection in S-DNN boosts enhancement performance compared to the DNN methods. The LSTM obtains further improvement by incorporating the past and current temporal information. The utilization of past, current and future temporal information in BLSTM shows advantages over the LSTM and DNN based method. The CRN method incorporates the convolutional encoder-decoder with the LSTM. The convolutional encoderencoder takes advantage of the convolutional layer and batch normalization to provide a high-level representation of the input feature, which improves the enhancement performance. Incorporating of the BLSTM layers, the CRBN offers higher improvements over the CRN method in terms of $\triangle \mathrm{SDR}$, STOI and PESQ. The GRN method uses gated linear units to control the information flow, and dilated convolutional layers to expand the receptive fields. These strategies enable the GRN method to outperform the methods above.

The proposed MCGN method provides the highest improvements over all the baseline methods in terms of $\triangle \mathrm{SDR}$, STOI and PESQ. The t-test results in Table IV also show that the improvement of the proposed MCGN methods is statistically significant.

The proposed MCGN method provides the highest improvements over all the baseline methods in terms of $\Delta$ SDR, STOI and PESQ. The t-test results in Table IV also show that the improvement of the proposed MCGN methods is statistically 
TABLE V

SPEECH ENHANCEMENT PERFORMANCE COMPARISONS IN TERMS OF STOI AND PESQ OVER THREE DIFFERENT TYPES OF SEEN NOISES WITH DIFFERENT BASELINE METHODS AND SNR LEVELS. EACH RESULT IS THE AVERAGED VALUE OF 500 EXPERIMENTS. Italic TEXT REFERS TO THE PROPOSED METHOD. BOLD NUMBER INDICATES THE BEST PERFORMANCE.

\begin{tabular}{|c|c|c|c|c|c|c|c|c|c|c|c|c|}
\hline Measure & \multicolumn{12}{|c|}{ STOI (\%) } \\
\hline Noises & \multicolumn{4}{|c|}{ Babble } & \multicolumn{4}{|c|}{ Leopard } & \multicolumn{4}{|c|}{ F16 } \\
\hline SNR & $-5 \mathrm{~dB}$ & $0 \mathrm{~dB}$ & $5 \mathrm{~dB}$ & Avg. & $-5 \mathrm{~dB}$ & $0 \mathrm{~dB}$ & $5 \mathrm{~dB}$ & Avg. & $-5 \mathrm{~dB}$ & $0 \mathrm{~dB}$ & $5 \mathrm{~dB}$ & Avg. \\
\hline Noisy Mixture & 56.63 & 66.36 & 75.28 & 66.09 & 73.22 & 78.06 & 82.26 & 77.85 & 57.4 & 66.83 & 75.61 & 66.61 \\
\hline LSTM [16] & 70.30 & 78.18 & 83.96 & 77.48 & 81.15 & 84.76 & 87.45 & 84.45 & 74.21 & 80.91 & 85.23 & 80.12 \\
\hline BLSTM [15] & 71.24 & 78.86 & 84.53 & 78.21 & 82.02 & 85.39 & 87.99 & 85.13 & 74.98 & 81.28 & 85.93 & 80.73 \\
\hline CRN [22] & 71.34 & 79.18 & 84.67 & 78.40 & 82.30 & 85.77 & 88.17 & 85.41 & 75.06 & 81.98 & 86.13 & 81.06 \\
\hline GRN [15] & 73.53 & 80.06 & 84.29 & 79.27 & 82.77 & 85.9 & 88.21 & 85.63 & 77.38 & 82.66 & 86.11 & 82.05 \\
\hline$M C G N$ & 78.46 & 83.19 & 86.97 & 82.87 & 85.01 & 87.45 & 89.45 & 87.30 & 79.56 & 84.27 & 87.64 & 83.82 \\
\hline Measure & \multicolumn{12}{|c|}{ PESQ } \\
\hline Noises & \multicolumn{4}{|c|}{ Babble } & \multicolumn{4}{|c|}{ Leopard } & \multicolumn{4}{|c|}{ F16 } \\
\hline SNR & $-5 \mathrm{~dB}$ & $0 \mathrm{~dB}$ & $5 \mathrm{~dB}$ & Avg. & $-5 \mathrm{~dB}$ & $0 \mathrm{~dB}$ & $5 \mathrm{~dB}$ & Avg. & $-5 \mathrm{~dB}$ & $0 \mathrm{~dB}$ & $5 \mathrm{~dB}$ & Avg. \\
\hline Noisy Mixture & 1.38 & 1.65 & 1.96 & 1.66 & 1.77 & 2.09 & 2.37 & 2.08 & 1.31 & 1.55 & 1.83 & 1.56 \\
\hline LSTM [16] & 1.88 & 2.25 & 2.56 & 2.23 & 2.44 & 2.69 & 2.92 & 2.68 & 2.02 & 2.37 & 2.63 & 2.34 \\
\hline BLSTM [15] & 1.91 & 2.29 & 2.60 & 2.26 & 2.50 & 2.75 & 2.98 & 2.74 & 2.10 & 2.41 & 2.67 & 2.39 \\
\hline CRN [22] & 1.93 & 2.31 & 2.61 & 2.28 & 2.53 & 2.79 & 3.00 & 2.77 & 2.14 & 2.45 & 2.69 & 2.43 \\
\hline GRN [15] & 2.03 & 2.33 & 2.62 & 2.33 & 2.53 & 2.80 & 3.01 & 2.78 & 2.20 & 2.47 & 2.70 & 2.46 \\
\hline$M C G N$ & 2.27 & 2.55 & 2.77 & 2.53 & 2.71 & 2.93 & 3.10 & 2.91 & 2.35 & 2.60 & 2.81 & 2.59 \\
\hline
\end{tabular}

significant.

\section{E. Experiments on Published Dataset}

We also evaluate the proposed MCGN method on the second dataset that we mentioned earlier, i.e. the published dataset generated by the VCTK corpus. Fig. 5 shows experimental results. Note that the model size (i.e. the number of parameters) of SEGAN, Wavenet and the proposed MCGN is $193 \mathrm{M}$, 34.3M, 64M respectively. The no-casual, dilated convolutions controlled by the Sigmoid gate in every layer help to enlarge the receptive fields of every kernel, and thus to utilize the interdependency among input features. The future samples help the Wavenet to perform better. Our MCGN method produces substantially better enhancement performance, since the MCFR model provides weighted multi-scale feature in every layer, and captures the interdependency among different frames including future frames.
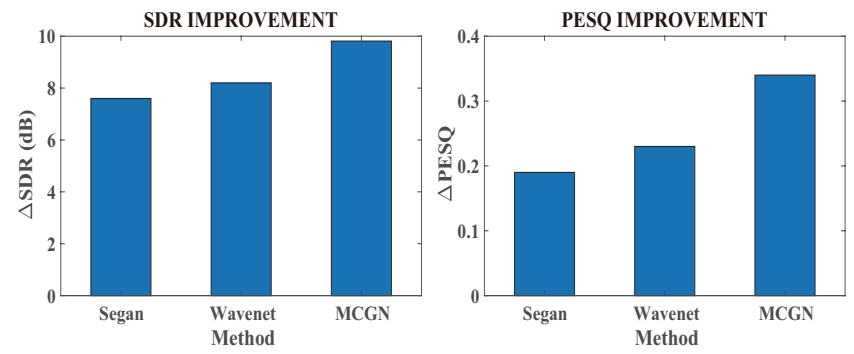

Fig. 5. Speech enhancement comparison in terms of $\triangle \mathrm{SDR}$ and $\triangle \mathrm{PESQ}$ for SEGAN [23], Wavenet [21] and the proposed MCGN. The enhancement results are the averaged value of 824 noisy mixtures.

\section{F. Additional Experiments}

Figs. $6 \& 7$ and Tables V \& VI provide experimental results in terms of $\triangle \mathrm{SDR}$, STOI and PESQ for the proposed MCGN and four baseline methods (i.e. LSTM, BLSTM, CRN and GRN) with seen and unseen noises, for the larger dataset (i.e. 50000 training signals and 2500 testing signals for each SNR level, described in Section III.A).

It can be observed that the proposed MCGN method performs better than all the baseline methods, and shows
TABLE VI

SPEECH ENHANCEMENT PERFORMANCE COMPARISONS IN TERMS OF STOI AND PESQ OVER TWO DIFFERENT TYPES OF UNSEEN NOISES WITH BASELINE METHODS AND SNR LEVELS. EACH RESULT IS THE AVERAGED VALUE OF 500 EXPERIMENTS. Italic TEXT REFERS TO THE PROPOSED METHOD. BOLD NUMBER INDICATES THE BEST PERFORMANCE.

\begin{tabular}{|c|c|c|c|c|c|c|c|c|}
\hline Measure & \multicolumn{8}{|c|}{ STOI (\%) } \\
\hline Noises & \multicolumn{4}{|c|}{ N56 } & \multicolumn{4}{|c|}{ N72 } \\
\hline SNR & $-5 \mathrm{~dB}$ & $0 \mathrm{~dB}$ & $5 \mathrm{~dB}$ & Avg. & $-5 \mathrm{~dB}$ & $0 \mathrm{~dB}$ & $5 \mathrm{~dB}$ & Avg. \\
\hline Mixture & 57.83 & 67.19 & 76.02 & 67.01 & 79.19 & 78.37 & 83.44 & 80.33 \\
\hline LSTM [16] & 77.29 & 82.25 & 85.93 & 81.82 & 81.75 & 85.62 & 88.36 & 85.24 \\
\hline BLSTM [15] & 77.59 & 82.54 & 86.45 & 82.19 & 82.59 & 86.18 & 89.11 & 85.96 \\
\hline CRN [22] & 77.90 & 83.62 & 87.24 & 82.92 & 82.61 & 86.81 & 89.25 & 86.22 \\
\hline GRN [15] & 78.50 & 83.88 & 87.56 & 83.31 & 83.00 & 87.4 & 89.35 & 86.58 \\
\hline$M C G N$ & 83.87 & 87.20 & 89.67 & 86.91 & 85.57 & 88.55 & 90.54 & 88.22 \\
\hline Measure & \multicolumn{8}{|c|}{ PESQ } \\
\hline Noises & \multicolumn{4}{|c|}{ N56 } & \multicolumn{4}{|c|}{ N72 } \\
\hline SNR & $-5 \mathrm{~dB}$ & $0 \mathrm{~dB}$ & $5 \mathrm{~dB}$ & Avg. & $-5 \mathrm{~dB}$ & $0 \mathrm{~dB}$ & $5 \mathrm{~dB}$ & Avg. \\
\hline Mixture & 1.20 & 1.33 & 1.56 & 1.36 & 1.57 & 1.84 & 2.10 & 1.84 \\
\hline LSTM [16] & 2.03 & 2.22 & 2.40 & 2.22 & 2.15 & 2.39 & 2.58 & 2.37 \\
\hline BLSTM [15] & 2.05 & 2.26 & 2.44 & 2.25 & 2.23 & 2.42 & 2.62 & 2.42 \\
\hline CRN [22] & 2.07 & 2.31 & 2.47 & 2.28 & 2.28 & 2.46 & 2.65 & 2.46 \\
\hline GRN [15] & 2.08 & 2.32 & 2.45 & 2.28 & 2.29 & 2.46 & 2.67 & 2.47 \\
\hline$M C G N$ & 2.27 & 2.51 & 2.64 & 2.47 & 2.42 & 2.61 & 2.82 & 2.62 \\
\hline
\end{tabular}

similar trends as for the smaller dataset tested earlier. All the methods provide some improvements over the noisy mixtures, which indicate that they are effective for speech enhancement with seen and unseen noises. The BLSTM provides more improvements than LSTM, since it uses additional information from the future frames, in contrast to the information from only current and previous frames used in LSTM. The CRN uses the CED to capture local T-F patterns from input noisy mixtures, also uses the LSTM layers to relate the past frames with current frames, thus offering higher improvements than the LSTM and BLSTM. The GRN shows advantage over LSTM, BLTM and CRN, due to the employment of the dilated 2-D convolutional layers for expanding the receptive fields in the T-F domain, and the gated convolution to control the information flow between layers.

The proposed MCGN method employs multi-scale 2Dconvolutional layers to enlarge the receptive fields in the T$\mathrm{F}$ domain, as a result, the features extracted are in different scales, capturing both local and contextual information. The multi-scale feature is assigned with different weights to provide a better feature representation. Furthermore, the BGRU layers are utilized to model the interdependency among the 

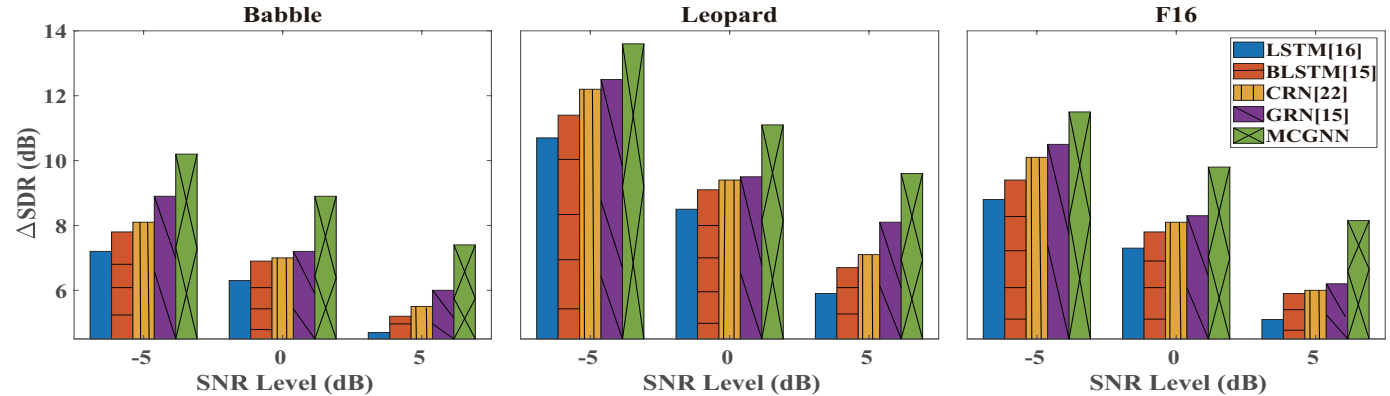

Fig. 6. Speech enhancement performance comparison in terms of $\Delta$ SDR for three types of noise with different methods and SNR levels. Each result is the averaged value of 500 experiments.
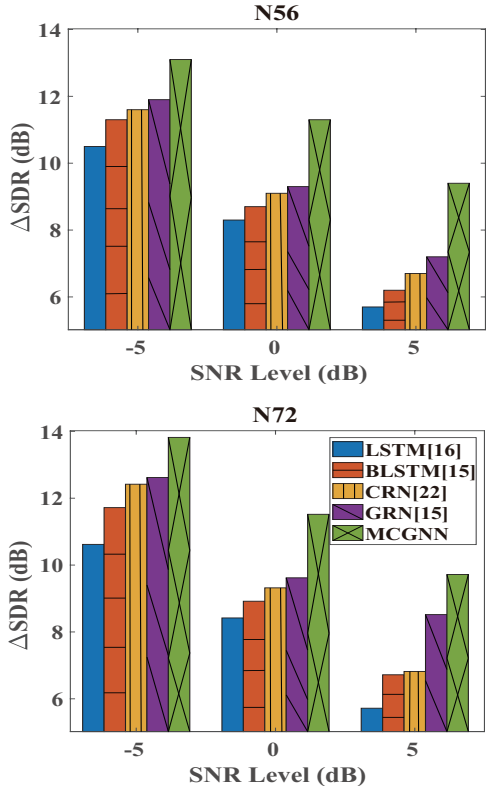

Fig. 7. Speech enhancement performance comparison in terms of $\Delta$ SDR for two unseen noises with different methods and SNR levels. Each result is the averaged value of 500 experiments.

past, current and future frames. In summary, these results further confirm that the MCGN outperforms baseline methods.

\section{G. Convergence Lines and Spectrums}

Fig. 8 demonstrates the testing MSEs of the baseline methods and the proposed MCGN and MCGN without multi-scale output (MCGN(NM)) layers over epochs. It can be seen that the MCGN converges faster than the baseline methods and reaches the lowest MSE. After 20 epochs training, the MCGN and MCGN(NM) offer similar MSEs, but the convergence speech of MCGN is faster than MCGN(NM) at 1-5 epochs. This suggests that the multi-scale feature representation may also help improve the convergence speed of the algorithm, apart from improving its enhancement performance.

We plot a set of spectrums in Fig. 9. It can be seen that the baseline methods and the proposed MCGN method provide different enhancement performance in terms of reconstruction of target speech. The spectrums of the proposed MCGN method are closer to the spectrums of the target speech, which again confirms that the MCGN outperforms the baseline methods.

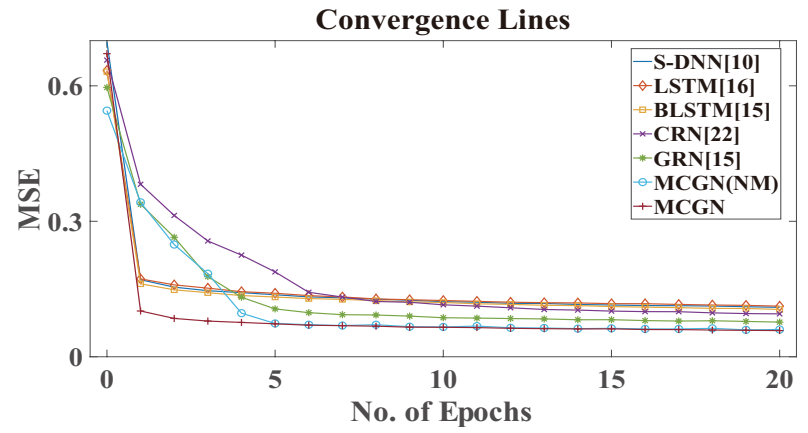

Fig. 8. Mean squared errors over training epochs for S-DNN, LSTM, BLSTM, CRN, GRN, MCGN and MCGN(NM) on the testing set. The MCGN(NM) represents the delete the multi-scale output layers, only use the normal output layer. All models are evaluated with a testing set of unseen speakers.

\section{H. Component Analysis}

We also conduct a series of experiments to investigate the efficiency of different components in the proposed model. In the component analysis, the ablation experiments are performed by removing different components to show how it affects the enhancement performance.

Table VII provides the experimental results of using various components in terms of the $\triangle$ SDR, STOI, PESQ and the number of parameters (million). Full means the full MCGN framework. No bottleneck represents removing the bottleneck layers in MCGN. No FC represents removing the fully connected layers in MCGN. No MCFR means using the single kernel in each encoder-decoder layer. No CL represents removing the connection layers that include a dense layer and two BGRU layers. No FR denotes removing feature recalibration, which means that the different scaled features use the same weight and are concatenated directly.

TABLE VII

COMPONENT ANALYSIS

\begin{tabular}{ccccc}
\hline \hline Measures & $\Delta$ SDR & STOI & PESQ & No. of Parameters \\
\hline Full & 10.20 & 81.40 & 2.40 & 64 \\
No Bottleneck & 10.39 & 81.60 & 2.43 & 120 \\
No FC & 10.24 & 81.51 & 2.40 & 112 \\
No CL & 9.27 & 77.25 & 2.23 & 42 \\
No MCFR & 9.12 & 77.42 & 2.20 & 28 \\
No FR & 9.61 & 79.87 & 2.32 & 56 \\
\hline \hline
\end{tabular}

The bottleneck layers employ fewer channels than previous layers to compress the information from previous convolutional layers, and this can reduce the computational cost with slight information loss, as shown in the experimental results. Unlike bottleneck layers in the convolutional encoder and 

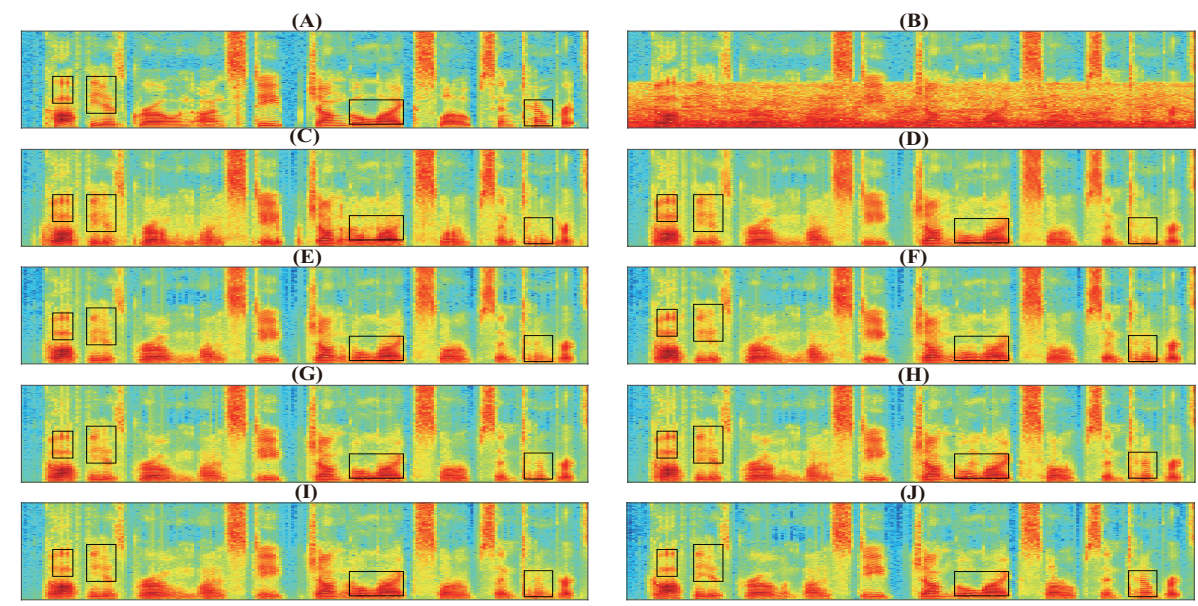

Fig. 9. Spectrums of different signals: (A) clean speech; (B) noisy speech mixture; (C) enhanced speech by S-DNN [10]; (D) enhanced speech by LSTM [16]; (E) enhanced speech by BLSTM [15]; (F) enhanced speech by the proposed MRCAE [20] (G) enhanced speech by the proposed CRN [22]; (H) enhanced speech by the proposed CRBN; (I) enhanced speech by the proposed GRN [15]. (J) enhanced speech by the proposed MCGN.

decoder, the FC layer with non-linear activation can produce a compact representation of the encoder output before the BGRU layer is applied. The bottleneck and FC layers help capture global information from the mixture. In addition, the interdependency among the past, current and future frames is captured by the BGRU layers. Therefore, the CL can employ BGRU and FC layers to provide improvements of enhancement performance and parameter efficiency. The results also show that the MCFR module can improve the performance by capturing the features in different scales using paralleled kernels of different size.

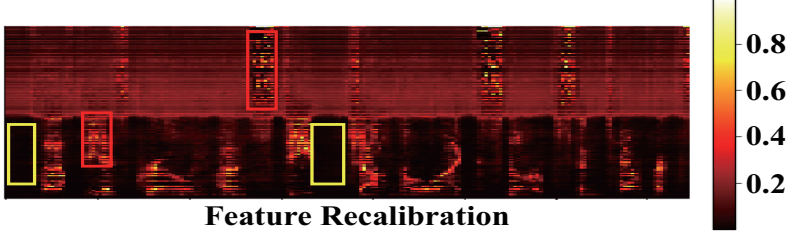

Fig. 10. The weights obtained by feature recalibration are shown as a hotmap, where the horizontal and vertical axis denote the time and frequency, respectively, and the color-bar shows the values of the weights.

Fig. 10 shows the weights obtained by feature recalibration in the last layer of the multi-scale decovolutional layer. The color-bar shows the weight values, and the deeper color represents a smaller value. Comparing Fig. 10 with Fig. 9 (A), (B), we can observe that the weights of high values capture the target speech very well. For example, the areas highlighted with the red blocks represent speech components, while those highlighted with yellow blocks represent components from noise. It can be observed that the feature recalibration tends to assign the features from speech with higher weights, and features from noise with lower weights. Therefore, the feature recalibration helps suppress noise and improve reconstruction of the target speech.

\section{Kernel Size Analysis}

We perform further experiments to analyse the relation between enhancement performance and kernel sizes with unseen noises. These experiments use kernel size varied from $1 \times 2$ to $11 \times 11$, thus exploiting different receptive fields in the T-F domain. Table VIII provides the experimental results in terms
TABLE VIII

KERNEL SIZE ANALYSIS

\begin{tabular}{cccc}
\hline \hline Filte Size & $\Delta$ SDR & STOI & PESQ \\
\hline $1 \times 2$ & 10.55 & 72.07 & 1.71 \\
$2 \times 2$ & 10.72 & 72.21 & 1.72 \\
$2 \times 3$ & 10.76 & 72.30 & 1.73 \\
$4 \times 5$ & 10.88 & 72.37 & 1.73 \\
$5 \times 5$ & 11.16 & 72.97 & 1.76 \\
$7 \times 7$ & 11.18 & 73.15 & 1.77 \\
$11 \times 11$ & 11.23 & 73.07 & 1.75 \\
Multi-Kernel & $\mathbf{1 1 . 7 2}$ & $\mathbf{7 6 . 2 1}$ & $\mathbf{1 . 9 2}$ \\
\hline \hline
\end{tabular}

of $\triangle$ SDR, STOI, and PESQ. The enhancement performances increase with the increase in the kernel size, e.g. from $1 \times 2$ to $7 \times 7$, but then starts to saturate for the further increase to $11 \times 11$. However, the performance difference is relatively small.

A larger kernel size, such as $7 \times 7$, can provide a larger receptive field, which generates the T-F feature map from a larger region i.e. contextual information, which may be effective in mitigating noise, and a smaller kernel size such as $1 \times 2$ captures the feature map among a smaller region i.e. local information, thus effective in retaining the detailed $\mathrm{T}$ F structure. This appears to be consistent with the analysis in [15], [22]. Unlike the BGRU layers which capture the interdependency among time frames (i.e. time-domain), the 2D-convolutional layers allow the expansion along both time and frequency.

As shown in Table VIII, the performance is also dependent on the choice of the kernel size. When the kernel size is larger than $7 \times 7$, performance may decrease in terms of STOI and PESQ. Using paralleled multi-kernel helps the model to capture the features in different scales, thus exploiting both local and contextual information, and to offer better enhancement performance with unseen noises, as in our proposed method.

To interpret the use of different kernel sizes, we have provided an example of the feature map obtained by using kernels of different sizes in the first multi-scale convolutional layer, as shown in Fig. 11, using kernels of size $1 \times 2,3 \times 4$, and $7 \times 7$. It can be seen, although the kernel at each scale extracted both speech and noise components, as shown in the regions highlighted with blue and black, the feature maps obtained 


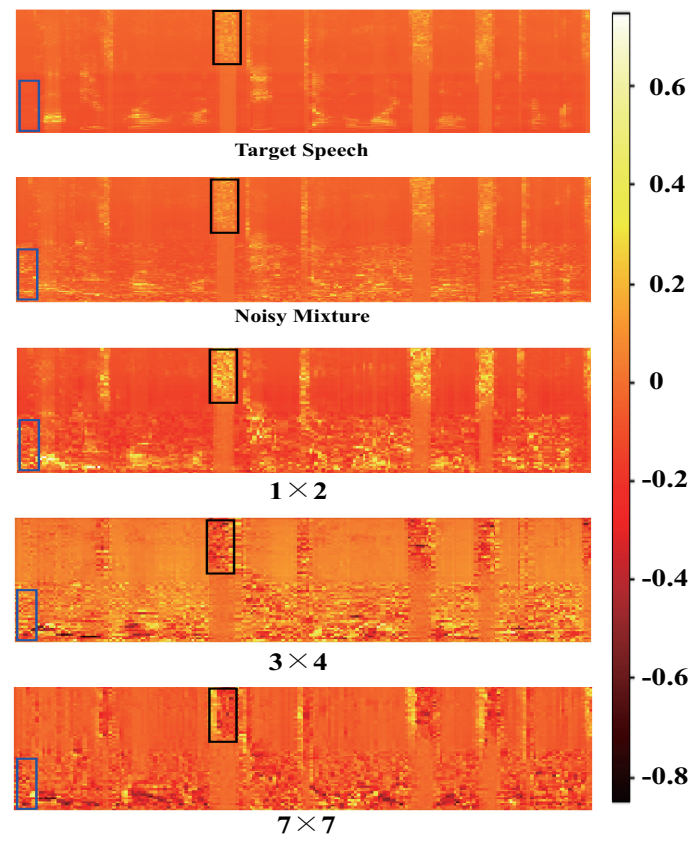

Fig. 11. Feature maps in MCGN with different kernel sizes. Also, the magnitude spectra of target speech and noisy mixture are provided. The horizontal and vertical axes denote the time and frequency, respectively. The color-bar shows the L2-normalized values. Example of components of speech and noise are highlighted with black and blue blocks, respectively.

with these kernels characterise different receptive fields, for example, with the large kernel, more heavy smoothing is applied which is effective in mitigating the impact of noise, while the use of a small kernel can retain the fine structure of the spectrum. Therefore, using a bank of kernels, the system has a better chance to capture and distinguish the features from speech and noise, thus further improves the speech enhancement performance.

\section{CONCLUSIONS}

We have presented a new framework for single channel speech enhancement. The proposed MCGN introduced several novel strategies to improve the enhancement performance and computational efficiency of the neural network algorithm. Firstly, we introduced the MCFR structure to extract the features in different scales, capturing both the local and contextual information from the speech mixtures. In addition, the feature recalibration network was implemented using a gating function to control the information flow, which assigns higher weights to speech components and lower weights to noise components, and thus improves the reconstruction of target speech by suppressing the noise from noisy mixtures. Secondly, we introduced the bottleneck convolutional and deconvolutional layers to reduce information flow dimension in encoder and decoder, but to retain the information. Thirdly, the efficiency connection module was introduced. The fully connected layer was used to reduce the dimension of the output of the convolutional encoder. The BGRU was exploited to capture the interdependency among the past, current and future temporal frames, which provides comparable performance with fewer parameters than BLSTM. Finally, we introduced the multi-scale convolutional output layer, then summed the multi-scale outputs to accelerate the convergence speed. A variety of noises were used to examine the enhancement performance of the system. The unseen speakers with the seen and unseen noises were exploited to evaluate the efficacy of the proposed method. The experimental results confirmed the improved performance of the proposed method overs the stateof-the-art baseline methods.

\section{ACKNOWLEDGEMENT}

The authors would like to thank the Associate Editor and the anonymous reviewers for their valuable input to improving the paper.

\section{REFERENCES}

[1] D. L. Wang, "Deep learning reinvents the hearing aid," IEEE Spectrum, vol. March Issue, pp. 32-37, 2017.

[2] P. C. Loizou, Speech Enhancement : Theory and Practice. CRC Press, 2013.

[3] S. M. Naqvi, M. Yu, and J. A. Chambers, "A multimodal approach to blind source separation of moving sources," IEEE Journal of Selected Topics in Signal Processing, vol. 4, pp. 895-910, 2010.

[4] Y. Sun, Y. Xian, W. W. Wang, and S. M. Naqvi, "Monaural source separation in complex domain with long short-term memory neural network," IEEE Journal of Selected Topics in Signal Processing, vol. 13, no. 2, pp. 359-369, 2019 .

[5] B. Rivet, W. W. Wang, S. M. Naqvi, and J. A. Chambers, "Audiovisual speech source separation: An overview of key methodologies," IEEE Signal Processing Magazine, vol. 31, no. 3, pp. 125 - 134, 2014.

[6] J. S. Lim and A. V. Oppenheim, "All-pole modeling of degraded speech," IEEE Transactions on Acoustics, Speech, and Signal Processing, vol. 26, no. 3, pp. 197-210, 1978F.

[7] Y. Ephraim and D. Malah, "Speech enhancement using a minimum-mean square error short-time spectral amplitude estimator," IEEE Transactions on Acoustics, Speech, and Signal Processing, vol. 32, no. 6, pp. 11091121,1984

[8] Y. X. Wang and D. L. Wang, "Towards scaling up classification-based speech separation," IEEE Transactions on Audio, Speech, and Language Processing, vol. 21, no. 7, pp. 1381-1390, 2013.

[9] K. Han, Y. X. Wang, D. L. Wang, W. S. Woods, I. Merks, and T. Zhang, "Learning spectral mapping for speech dereverberation and denoising," IEEE/ACM Transactions on Audio, Speech, and Language Processing, vol. 23, no. 6, pp. 189-198, 2015.

[10] M. Tu and X. X. Zhang, "Speech enhancement based on deep neural networks with skip connections," IEEE International Conference on Acoustics, Speech and Signal Processing (ICASSP), 2017.

[11] Y. Xu, J. Du, L. R. Dai, and C. H. Lee, "A regression approach to speech enhancement based on deep neural networks," IEEE Transactions on Audio, Speech, and Language Processing, vol. 23, no. 1, pp. 7-19, 2015.

[12] S. Rickard and O. Yilmaz, "On the approximate w-disjoint orthogonality of speech," IEEE International Conference on Acoustics, Speech and Signal Processing (ICASSP), 2002.

[13] Y. Jiang, D. L. Wang, R. Liu, and Z. Feng, "Binaural classification for reverberant speech segregation using deep neural networks," IEEE/ACM Transactions on Audio, Speech, and Language Processing, vol. 22, no. 12 , pp. 2112-2121, 2014.

[14] A. Narayanan and D. L. Wang, "Ideal ratio mask estimation using deep neural network for robust speech separation," IEEE International Conference on Acoustics, Speech and Signal Processing (ICASSP), 2013.

[15] K. Tan and D. L. Wang, "Gated residual networks with dilated convolutions for monaural speech enhancement," IEEE/ACM Transactions on Audio, Speech, and Language Processing, vol. 27, no. 1, pp. 189-198, 2019.

[16] J. T. Chen and D. L. Wang, "Long short-term memory for speaker generalization in supervised speech separation," The Journal of the Acoustical Society of America, vol. 141, no. 6, pp. 4705-4714, 2017.

[17] S. Hochreiter and J. Schmidhuber, Long Short-Term Memory. Neural Comput, 1997. 
[18] F. Weninger, H. Erdogan, S. Watanabe, E. Vincent, J. L. Roux, J. R. Hershey, and B. Schuller, "Speech enhancement with 1stm recurrent neural networks and its application to noise-robust asr," International Conference on Latent Variable Analysis and Signal Separation (LVA/ICA), 2015.

[19] S. R. Park and J. Lee, "A fully convolutional neural network for speech enhancement," Annual Conference of the International Speech Communication Association (INTERSPEECH), pp. 1993-1997, 2017.

[20] E. M. Grais, D. Ward, and M. D. Plumbley, "Raw multi-channel audio source separation using multi-resolution convolutional auto-encoders," Proc. of European Signal Processing Conference (EUSIPCO), 2018.

[21] D. Rethage, J. Pons, and X. Serra, "A wavenet for speech denoising," IEEE International Conference on Acoustics, Speech and Signal Processing (ICASSP), 2018.

[22] K. Tan and D. L. Wang, "A convolutional recurrent neural network for real-time speech enhancement," Annual Conference of the International Speech Communication Association (INTERSPEECH), 2018.

[23] P. Santiago, B. Antonio, and S. Joan, "Segan: Speech enhancement generative adversarial network," International Speech Communication Association (Interspeech), 2017.

[24] J. Y. Chung, C. Gulcehre, K. H. Cho, and Y. Bengio, "Empirical evaluation of gated recurrent neural networks on sequence modeling," Conference on Neural Information Processing Systems (NIPS), 2014.

[25] C. Szegedy, W. Liu, Y. Q. Jia, P. Sermanet, S. Reed, D. Anguelov, D. Erhan, V. Vanhoucke, and A. Rabinovich, "Going deeper with convolutions," Proc. of Computer Vision and Pattern Recognition (CVPR), 2015.

[26] V. Nair and G. E. Hinton, "Rectified linear units improve restricted boltzmann machines," International Conference on Machine Learning (ICML), 2010.

[27] A. L. Mass, A. Y. Hannun, and A. Y. Ng, "Rectifier nonlinearities improve neural network acoustic models," International Conference on Machine Learning (ICML), 2013.

[28] K. M. He, X. Y. Zhang, S. Q. Ren, and J. Sun, "Deep residual learning for image recognition," IEEE Conference on Computer Vision and Pattern Recognition (CVPR)), 2016.

[29] C. Szegedy, V. Vanhoucke, S. Ioffe, and Z. W. J. Shlens, "Rethinking the inception architecture for computer vision," Proc. of Computer Vision and Pattern Recognition (CVPR), 2016.

[30] K. Y. Cho, B. V. Merrienboer, C. Gulcehre, D. Bahdanau, F. Bougares, H. Schwenk, and Y. Bengio, "Learning phrase representations using rnn encoder-decoder for statistical machine translation," Conference on Empirical Methods in Natural Language Processing (EMNLP), 2014

[31] R. Jozefowicz, W. Zaremba, and I. Sutskever, "An empirical exploration of recurrent network architectures," International Conference on Machine Learning (ICML), 2014.

[32] J. S. Garofolo, L. F. Lamel, W. M. Fisher, J. G. Fiscus, D. S. Pallett, and N. L. Dahlgren, "Darpa timit acoustic phonetic continuous speech corpus cdrom," 1993

[33] G. N. Hu and D. L. Wang, "A tandem algorithm for pitch estimation and voiced speech segregation." IEEE Transactions on Audio, Speech, and Language Processing, vol. 18, no. 7, pp. 2067-2079, 2010.

[34] A. Varga and H. Steeneken, "Assessment for automatic speech recognition: II. NOISEX-92: A database and an experiment to study the effect of additive noise on speech recognition systems," Speech Commun, no. 12, pp. 247-251, 1993.

[35] C. Veaux, J. Yamagishi, and K. MacDonald, "Cstr vctk corpus: English multi-speaker corpus for cstr voice cloning toolkit," 2016.

[36] J. Thiemann, N. Ito, and E. Vincent, "The diverse environments multichannel acoustic noise database (demand): A database of multichannel environmental noise recordings," in Proc. 21st Int. Congr. on Acoustics, Montreal, Canada, Acoustical Society of America, 2013.

[37] E. Vincent, R. Gribonval, and C. Fevotte, "Performance measurement in blind audio source separation," IEEE Transanctions on Audio Speech and Language Processing, vol. 14, no. 4, pp. 1462-1469, 2006.

[38] Y. Hu and P. C. Loizou, "Evaluation of objective quality measures for speech enhancement," IEEE Transactions on Audio, Speech and Language Processing, vol. 16, no. 1, pp. 229-238, 2008.

[39] C. H. Taal, R. C. Hendriks, R. Heusdens, and J. Jensen, "An algorithm for intelligibility prediction of time frequency weighted noisy speech," IEEE Transactions on Audio, Speech, and Language Processing, vol. 19, no. 7, pp. 2125-2136, 2011.

[40] D. P. Kinqma and J. L. Ba, "Adam: A method for stochastic optimization," International Conference for Learning Representations (ICLR), 2015.

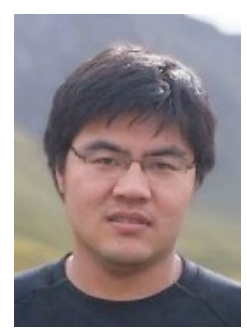

Yang Xian (Student Member, IEEE) received the B.Sc. degree in 2014, from the Zhengzhou University, China and M.Sc degree in 2016 from Newcastle University, U.K, respectively. He is currently pursuing the Ph.D. degree within Intelligent Sensing and Communications (ISC) Research Group Research Group, School of Engineering, Newcastle University, U.K. His research areas of interest include audio signal processing, speech source separation and enhancement based on deep learning.

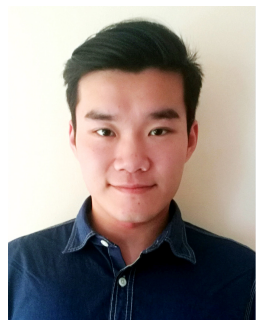

Yang Sun (Member, IEEE) received his Ph.D. degree within Intelligent Sensing and Communications (ISC) Research Group, School of Engineering, Newcastle University, U.K in 2019. Currently, Yang is a postdoctoral researcher at the Big Data Institute, University of Oxford, developing methods for brain lesion segmentation with multiple sclerosis. His research areas of interest include audio signal processing and biomedical image processing based on deep learning.

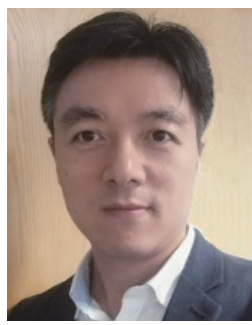

Wenwu Wang (Senior Member, IEEE) received the B.Sc. degree in 1997, the M.E. degree in 2000, and the $\mathrm{Ph} . \mathrm{D}$. degree in 2002, all from the College of Automation, Harbin Engineering University, China. $\mathrm{He}$ then worked in King's College London (20022003), Cardiff University (2004-2005), Tao Group Ltd. (now Antix Labs Ltd.) (2005-2006), Creative Labs (2006-2007), before joining University of Surrey in May 2007, where he is a Professor in Signal Processing and Machine Learning, and a CoDirector of the Machine Audition Lab within the Centre for Vision Speech and Signal Processing. He is also a Guest Professor at Qingdao University of Science and Technology, China. He was a Visiting Scholar at Ohio State University, USA, in 2008. His current research interests include blind signal processing, sparse signal processing, audio-visual signal processing, machine learning and perception, artificial intelligence, machine audition (listening), and statistical anomaly detection. He has (co)-authored over 250 publications in these areas.

Prof Wang is Senior Area Editor and was Associate Editor (2014-2018) for IEEE Transactions on Signal Processing. He is an Associate Editor for IEEE/ACM Transactions on Audio Speech and Language Processing, and an Associate Editor for EURASIP Journal on Audio Speech and Music Processing. He was a Publication Co-Chair for ICASSP 2019, Brighton, UK. He is a Member of the IEEE Signal Processing Theory and Methods Technical Committee and a Member of the IEEE Machine Learning for Signal Processing Technical Committee.

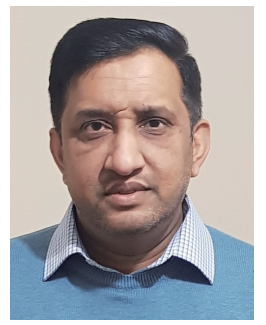

Syed Mohsen Naqvi (Senior Member, IEEE) received the Ph.D. degree in Signal Processing from Loughborough University, Loughborough, U.K., in 2009 and his Ph.D. thesis was on the EPSRC U.K. funded project. He was a Postdoctoral Research Associate on the EPSRC U.K.-funded projects and Research Excellence Framework (REF) Lecturer from 2009 to 2015. Prior to his postgraduate studies in Cardiff and Loughborough Universities U.K., he served the National Engineering and Scientific Commission (NESCOM) of Pakistan from 2002 to

2005.

Dr Naqvi is Associate Professor/Senior Lecturer in Signal and Information Processing at the School of Engineering, Newcastle University, Newcastle, U.K. He is leading Intelligent Sensing Lab at Newcastle University U.K. with major research focused on multimodal processing for human be-haviour analysis, multi-target tracking, and source separation all; for machine learning. $\mathrm{He}$ organized special sessions in FUSION, delivered seminars and was a speaker at UDRC Summer Schools 2015-2017. He has 150 publications with the main focus of his research being on audio-visual signal and information processing, machine learning and perception, reliable artificial intelligence, and action recognition and anomaly detection. $\mathrm{He}$ is an Associate Editor for Elsevier Journal on Signal Processing. He is Fellow of the Higher Education Academy. $\mathrm{He}$ is an Associate Editor for IEEE Transactions on Signal Processing. 